\title{
CRITERIOS DE ORGANIZACIÓN DE LA INFORMACIÓN AGROPECUARIA
}

\author{
GUILleRMo FlichmaN \\ Instituto Torcuato Di Tella
}

\section{INTRODUCCIÓN}

EN ESTAS PÁGINAS se intenta consignar algunas experiencias de trabajo con datos censales acerca de la estructura y el desarrollo agrario, y ciertas reflexiones metodológicas sobre las implicaciones que tienen las distintas perspectivas de abordar el problema.

Aunque pueda parecer excesivamente obvio, no está de más enfatizar la importancia de los estudios de la estructura agraria en relación con la dinámica demográfica. Sin embargo, tal como habría de surgir de la "evaluación" de los trabajos realizados acerca del tema, es bastante poco lo que se ha producido en este sentido en América Latina. En buena medida esto puede atribuirse a los criterios teóricos que han orientado, de manera implícita o explícita, los trabajos realizados.

Comenzaremos por delimitar cuáles son los problemas específicos con que nos enfrentamos cuando deseamos abordar el tema de "lo agrario", para de allí plantear el marco teórico en el cual consideramos deben abordarse los estudios empíricos.

Si se parte de un análisis en el que se consideran dominantes las relaciones de producción de tipo capitalista, en la esfera de producción agropecuaria juega un papel bien diferenciado un medio de producción no producido, limitado y no reproducible (relativamente), que es la tierra. La propiedad de la tierra permite, en determinado tipo de situaciones, la apropiación por parte de los terratenientes, propietarios de tierras, de una porción de ganancias extraordinarias que de ese modo se convierten en renta del suelo.

El problema de caracterizar de qué tipo de renta se trata en cada caso, y de efectuar algún tipo de medición de la misma, resulta muy importante como elemento que ayuda a la caracterización correcta de la clase de los propietarios de tierras, los terratenientes: la existencia de la renta, como forma de ingreso de la clase terrateniente, constituye algo "específico" por decirlo así, de lo agrario. El hecho de poder distinguir, dentro de la masa de ganancias percibidas por un terrateniente capitalista, por ejemplo, qué parte está constituida por beneficio capitalista y 
cuál parte por renta, permite efectuar una más ajustada caracterización de la clase de ese personaje.

Este hecho no presenta un interés sólo de tipo teórico, tiene importancia práctica para poder comprender la formación de distintos tipos de bloques dentro de los propietarios agrícolas ante medidas concretas de política económica que los afectan en forma discriminatoria según sean predominantemente "rentistas" o "capitalistas"."

Planteada la cuestión de la importancia que tiene la determinación de la magnitud relativa de la renta, en diferentes situaciones y para distintos tipos de explotaciones, se deja para un punto posterior de esta exposición, cuáles son los datos que se pueden encontrar que permitan valorar cuantitativamente este problema.

Otra cuestión de importancia fundamental que debe tener especial consideración en los estudios acerca de problemas agrarios, es la particularidad que presenta el medio rural, en cuanto a que en él coexisten de manera normal, aún en los países capitalistas más desarrollados, una gama de diferentes relaciones de producción. Si bien aparecen subordinadas al modo de producción capitalista, en la medida en que éste es el dominante, plantean una cantidad de problemas específicos que es de gran importancia analizar con detenimiento.

Por una parte, corresponde plantearse un primer interrogante fundamental ¿la existencia de relaciones de producción no capitalistas en el campo implica por necesidad una traba para el desarrollo de las fuerzas productivas, en una formación social donde el modo de producción capitalista es hegemónico ${ }^{2}$ Es difícil considerar que pueda existir una respuesta unívoca a esta pregunta, sin efectuar relaciones concretas con determinadas situaciones históricas en cada país que se considere. Pueden darse perfectamente casos en los que la expansión del capitalismo en el contexto internacional, promuevan el incremento y aun la creación de relaciones no-capitalistas de producción en determinados lugares y para ciertos tipos de producción. Éste fue el caso, por ejemplo, de las plantaciones algodoneras con mano de obra esclava en el sur norteamericano del siglo XIX. En el caso de la Argentina, las formas de arrendamiento en especie, con cláusulas contractuales no típicamente capitalistas, que se desarrollaron muchísimo en el período de gran expansión agrícola durante los primeros decenios de este siglo, también estuvieron totalmente ligadas con las necesidades de expansión del comercio internacional de productos alimenticios, que contribuía a acelerar el proceso de acumu-

\footnotetext{
1 En Argentina, actualmente se puede observar con claridad la oposición de los sectores más "terratenientes" a los impuestos a la tierra recién implantados, que son aceptados sin reservas por los más "capitalistas".

2 Varios estudios donde se muestre la funcionalidad de las relaciones precapitalistas de producción en sociedades dominadas por el modo de producción capitalista, son citados y sintetizados por Héctor Pérez Brignoli y Ciro Flamarión Santana Cardoso, Las clases sociales en el campo. Teoría y su aplicación al caso de Centroamérica. I. "Problemas teóricos y metodológicos", San José, Costa Rica, 1972.
} 
lación del capital en los países capitalistas europeos, en especial en Inglaterra. No sólo no frenaron, sino que expandieron de manera inusitada la producción durante un largo período. Fueron formas precapitalistas desarrolladas en virtud de la expansión del capitalismo como sistema mundial.

Señalar entonces la coexistencia de distintos tipos de relaciones de producción es algo muy conocido; lo que interesa destacar es que en la producción agraria esta coexistencia tiene una permanencia considerablemente mayor que en la industria. Por eso, reviste interés especial el análisis concreto de los distintos tipos de relaciones de producción, y los efectos que producen; así como las características diferentes de las clases o sectores de clase que aparecen en cada caso.

Si bien es importante el tipo de observación a que se hace referencia antes, en el sentido de poder comprender la aparición, desarrollo y supervivencia de relaciones de tipo precapitalista, o con más rigor, "no capitalista", en un contexto dominado por el modo de producción capitalista, esto no basta. No basta, porque es necesario explicar en forma particularizada el por qué de la "funcionalidad" de esas relaciones no capitalistas, cuál es la razón, o las razones que permiten la expansión de las fuerzas productivas en esas condiciones, por qué no frenan el proceso de acumulación del sistema en su conjunto. Nada de esto es evidente por sí mismo.

El hecho de que el ritmo de acumulación no se vea frenado por el mantenimiento de relaciones de producción no-capitalistas, debemos entenderlo en vinculación con las características del proceso de trabajo en la esfera de producción agrícola, comparado con lo que sucede en el caso de la producción industrial. El sistema de organización de la producción, en el caso de la agricultura no requiere en muchos casos (no en todos) de la producción en gran escala como condición necesaria de aumentar al máximo la eficiencia productiva. Esto hace que una empresa agrícola, basada en el trabajo familiar del campesino y que cuenta con un alto nivel de equipamiento pueda realizar perfectamente las tareas agrícolas con el mismo nivel de eficiencia técnica (desde el punto de vista de utilizar de manera correcta los recursos disponibles para producir) que una gran empresa capitalista.

Sin duda, esto no sucede con frecuencia en la industria. Fenómenos de esta naturaleza aparecen sólo en algunas ramas poco importantes, donde subsiste una suerte de "trabajo a domicilio" muy semejante al que describía Marx en El Capital y con un alto nivel de eficiencia (sería el caso de las tejedoras mecanizadas, por ejemplo). En la producción agropecuaria es diferente. Gran parte de la producción de cereales en Argentina, por ejemplo, está controlada por "empresas familiares mecanizadas" del tipo descrito. Aun en los Estados Unidos tienen también una considerable importancia.

Este es sólo uno de los tipos de relaciones de producción no capita- 
listas en sentido estrictd que existe; pero lo destacamos por el hecho de que se desarrolla en forma compatible con el mayor grado de avance de la tecnología agropecuaria, lo que no permite que sea considerado como un producto del atraso relativo del campo respecto de las actividades industriales. Este campesino tipo farmer nada tiene que ver con los campesinos parcelarios descritos por Marx en El Capital. ${ }^{3}$ Sin embargo, tampoco es un capitalista, ya que continúa siendo un productor directo, que refuerza su trabajo mediante el empleo de maquinarias, pero que no emplea trabajo asalariado, o lo hace sólo en forma subsidiaria.

No es nuestro propósito hacer aquí una descripción de las relaciones de producción en el campo, o de las clases sociales. Sólo se señalan algunos problemas que nos han guiado en la metodología seguida en las investigaciones empíricas.

El punto desarrollado antes tiene, por ejemplo, una importancia particular si se intentan estudiar los movimientos de población originados en áreas rurales. El grado de "población excedente" que puede generar un proceso de cambio tecnológico en el agro que implique mecanización, será mucho mayor en un área ocupada por empresas capitalistas que emplean trabajadores asalariados, que en otra donde predominen empresas campesinas de tipo familiar. Valga esta observación a título de ejemplo. En un estudio realizado hace algún tiempo ${ }^{4}$ se puede observar que, sin demasiadas diferencias en las condiciones tecnológicas, la densidad de población ocupada en el caso de las empresas campesinas basadas en el trabajo familiar era muchísimo más alta que en el resto. Una empresa familiar, cuyo objetivo es satisfacer las necesidades de consumo del grupo familiar, tolera un grado de subempleo en sus miembros mucho mayor que una empresa capitalista que busque maximizar sus ganancias.

Aparece entonces, con bastante claridad, la necesidad de determinar los distintos tipos de relaciones de producción que existen, su peso respecto de las distintas actividades, la tecnología que se emplea, la ocupación que existe en cada caso, etc. El objetivo central que nos guía para definir entonces métodos de trabajo con información censal agropecuaria, es definir criterios que permitan distinguir esos distintos tipos de relaciones de producción.

A continuación se hace una descripción somera de lo que constituye de manera habitual el material informativo disponible, luego se discuten las limitaciones de los trabajos que con esa "materia prima" se pueden realizar, para pasar después a efectuar una propuesta de trabajo basada en una construcción de los datos adecuada al objetivo que se persigue.

3 Carlos Marx, El capital, tomo III, México, Fondo de Cultura Económica, pp. 744-747, 1959 .

4 Guillermo Flichman y Lía Gens, "Caracterización socioeconómica del departamento de Cruz Alta", Buenos Aires, cEUR, publicación interna, 1972. 
II. Características de La información COMÚN disponible de oriGEN CENSAL Y TRABajOS REALIZABLES CON ELLA

En casi todos los países se efectúan con cierta regularidad relevamientos censales agropecuarios, en los cuales se reúne información básica bastante completa acerca de todo lo referente a la actividad agraria. En este trabajo no tiene mucho sentido comentar con amplitud el tipo de datos que se incluyen. El problema fundamental no radica tanto en este aspecto, sino en los criterios conforme a los cuales se organiza la información.

El dato con el cual se encuentra el investigador, no es el mismo consignado en la boleta censal, es el producto de todo un proceso de manipulación que culmina en una serie de cuadros tabulados con determinados criterios de agregación. Esto es, la producción del dato comienza con la confección del formulario censal y termina con la impresión de cuadros con datos. Quien va a trabajar con esos cuadros, se encuentra con una materia prima semielaborada que, desde un principio, acota sus posibilidades de producción a límites bastante precisos.

Sobre el problema inicial del contenido de la pregunta y cómo se plantea la pregunta, no entraremos en detalle. Sólo se considera conveniente señalar lo que suele suceder: la unidad de información censal, por lo común está constituida por la "explotación", definida en cuanto unidad física, geográfica, de explotación y no por la empresa, que en muchos casos puede estar constituida por un conjunto de "explotaciones". Esta cuestión provoca dificultades en el análisis de la distribución de la propiedad, y en la caracterización de las empresas rurales de tipo

- capitalista, y terrateniente-capitalista, que resultan subestimadas en tamaño y sobreestimadas en número.

Sin embargo, lo que más interesa es el problema de la elaboración de los datos, desde el formulario censal hasta los tomos impresos con los cuadros estadísticos. ${ }^{5}$

5 Respecto de este problema, Lenin hacía el siguiente comentario, que por su vigencia actual resulta de real interés citar en este trabajo: "Los censos alemanes, como todos los europeos del mismo género (a diferencia de los rusos) se basan en los informes recogidos sobre cada establecimiento agrícola por separado. La cantidad de informaciones reunidas suele aumentar con cada censo. ...La información que se obtiene de este modo sobre cada hacienda es por completo suficiente para la caracterización político-económica de las haciendas. Todo el problema, toda la difictultad de la tarea radica en resumir estos datos de modo tal que se pueda obtener una exacta caracterización político-económica de los diferentes grupos o tipos de hacienda en conjunto. Si el resumen es no satisfactorio, si la clasificación es equivocada o deficiente, puede darse un resultado tal $-\mathrm{y}$ se da siempre en la elaboración de los censos actuales-, que datos magníficos, con extraordinario detalle, relativos a cada establecimiento por separado, desaparecen, se pierden, se esfuman, cuando se trata de los millones de haciendas del país entero". V. I. Lenin, Vol. I, El régimen capitalista de la agricultura contemporá- 
En principio, el problema elemental es que los datos de miles y miles de "explotaciones" deben sumarse según algún criterio. En el peor de los casos, como es el de Argentina, se suman los datos de todas las variables consideradas en el censo, por unidades geográficas (departamentos). Esto permite saber, por ejemplo, cuánto trigo hay en cada departamento, cuántos arrendatarios, cuántos obreros y cuántos tractores, pero todo por separado. No se puede saber, por ejemplo, cuántas empresas tienen obreros asalariados, ni si las que más obreros tienen son las que se dedican de preferencia a tal o cual actividad, o son las más mecanizadas, o cualquiera otra cosa. Como se puede apreciar, la limitación de los análisis que se pueden hacer con ese tipo de datos es realmente impresionante. Dijimos que esto sucedía en el peor de los casos. En los mejores, se suele cruzar parte de la información con una clasificación de las explotaciones según su tamaño. Ésta es una vieja tradición estadística que ya existía en los censos agrarios norteamericanos y europeos de fines del siglo pasado y comienzos del presente. La limitación de los análisis basados en este criterio, es que por necesidad se mezclan tipos muy diferentes de empresas en cada estrato, tanto por las diferencias en las actividades realizadas como por las distintas relaciones de producción que pueden coexistir en los mismos estratos de tamaño. De todos modos es una forma de presentación de la información que permite la realización de análisis valiosos, si se parte de una tipología basada en delimitaciones dadas por las relaciones de producción y se realizan inferencias a partir de los estratos por tamaño. ${ }^{6}$

En resumen, se toma la información ya agrupada en estratos de tamaño y se observa, en el conjunto de cada estrato, cuál es, por ejemplo, el peso de los trabajadores asalariados sobre el total. En función de este dato, y de otros, referidos al nivel de "equipamiento" disponible, se pasa a caracterizar el conjunto de las explotaciones de cada estrato de una determinada manera. Esto implica siempre la inclusión de explotaciones que no responden a la caracterización media del estrato, y alteran en alguna medida la validez de las conclusiones del análisis.

A partir también de datos basados en tabulaciones por estratos de tamaño, se han realizado estudios acerca de la estructura agraria de América Latina en siete países por el Comité Interamericano de Desarrollo Agrícola (CIDA) ${ }^{7}$ En estos trabajos, el criterio para agrupar las explotaciones agropecuarias está basado en la posibilidad de ocupación de mano de obra por tamaño, según región que se considere. Los autores

nea, 1910; publicado en Obras Completas, Buenos Aires, Editorial Cartago, tomo XVI, 1960.

6 Lenin, en el trabajo citado, hace precisamente una elaboración con esas características.

7 Comité Interamericano de Desarrollo Agrícola, Tenencia de la tierra y desarrollo socioeconómico del sector agrario. Estos estudios fueron realizados para Argentina, Brasil, Colombia, Chile, Ecuador, Guatemala y Perú. El correspondiente a Argentina fue publicado por la Unión Panamericana, Washington, sin fecha. 
lo explican de la siguiente manera: "La extensión física absoluta de las fincas difícilmente permite hacer una comparación entre ellas en regiones de diferentes climas, suelos, mercados y niveles de desarrollo técnico y económico. Ella no es una medida satisfactoria ni" siquiera para comparar las fincas que se encuentran dentro de una región pequeña, si es que hay gran diferencia entre la calidad de sus suelos o el acceso a los mercados. A fin de reducir esta dificultad, en el presente análisis y para fines de clasificación se ha introducido un concepto de tamaño basado en la extensión de tierra necesaria para proveer de empleo remunerativo a una familia campesina típica, usando los recursos técnicos prevalecientes en la región y de acuerdo a los valores culturales locales ..."

"... En base a los datos disponibles" ... "se ha estimado la distribución de las fincas por tamaño en cuatro grupos a saber:

1. Tamaño subfamiliar, cuyas tierras son insuficientes para satisfacer las necesidades mínimas de una familia y para permitir la utilización productiva de su trabajo durante todo el año.

2. El tamaño familiar, es el que dispone de suficiente tierra para mantener una familia a un nivel satisfactorio de vida mediante el trabajo de sus miembros y la aplicación de la técnica predominante en el área.

3. El tamaño multifamiliar mediano, es el que tiene las tierras necesarias para emplear un número mayor de trabajadores que el que aportan los miembros de la familia; pero no tantos como para requerir una organización jerárquica a base de la designación de un administrador, mayoral, etc.

4. El tamaño multifamiliar grande, es el que posee tierras suficientes para dar ocupación permanente a una fuerza de trabajo mucho mayor que los miembros de la familia de su conductor y dentro de cuya unidad sí se requiere la división del trabajo y la organización jerárquica". ${ }^{8}$

Como ya se dijo, este tipo de clasificación parte también de datos agrupados por tamaño. Pero los reagrupa, no a partir de la importancia del trabajo asalariado, criterio básico si se quieren tener en cuenta las relaciones de producción, sino de la relación tamaño-ocupación en cada región que se considere. El eje ideológico de esta tipología es la "unidad económica" o "explotación familiar", que permite cortar hacia abajo y hacia arriba.

Existen distintos problemas con este tipo de categorización. Por una parte, si coincidiera el tamaño familiar de una explotación, con el hecho de que ésta sea explotada de manera efectiva por una empresa de tipo familiar, econtraríamos una situación semejante a la descrita antes. Esto es, se podría suponer que las subfamiliares implican campesinos semiproletarios, las familiares campesinos medios, las multifamiliares medianas campesinos ricos semicapitalistas y las multifamiliares grandes empresas capitalistas agrarias. Sin embargo, esto no es del todo cierto, ya que

8 Comité Interamericano de Desarrollo Agrícola, op. cit., p. IV y V. 
existen muchas explotaciones agrarias, que por su tamaño ocupan, por ejemplo, tres personas permanentes, quedando clasificadas como "familiares" en esta tipología y que pueden ser totalmente capitalistas, si esas tres personas son asalariadas.

El otro problema es el de que en una zona donde predomina la ganadería, por ejemplo, si hay algunas explotaciones agrícolas de un tamaño correspondiente a ganaderas subfamiliares, pero que por ser más intensivas son, por ejemplo, familiares, quedarán sin embargo clasificadas como subfamiliares por ser la ganadería lo dominante en esa zona.

El efecto de estos inconvenientes puede verse en el hecho siguiente: en las explotaciones subfamiliares, para toda Argentina, aparecen trabajadores asalariados en $15 \%$ del total de mano de obra en este tipo de explotaciones. En las familiares un $33 \%$. Esto significa que hay mezcla de explotaciones que usan trabajadores asalariados dentro de categorías en las que supuestamente no tendría que haber. Dentro del total de asalariados, los correspondientes a las explotaciones "familiares" y "subfamiliares" son nada menos que $57 \%$ del total. ${ }^{9}$

$\mathrm{Al}$ resumir lo anterior, se puede decir que el sistema de categorización del Comité Interamericano de Desarrollo Agrícola (CIDA), presenta problemas conceptuales, por los tipos que define y problemas de errores necesarios de clasificación, por la heterogeneidad de explotaciones de un mismo estrato, aun dentro de una misma zona. Por lo que vimos en el caso de la mano de obra asalariada, los problemas son bastante graves.

Tal vez en su momento resultaba imposible planear una tabulación censal con criterios diferentes. Pese a las limitaciones señaladas, hasta el presente los trabajos del ciDA constituyen una de las principales y mejores fuentes para los estudios que sobre estructura agraria se han hecho en nuestros países, usando datos de origen censal.

Otro tipo de trabajo que puede realizarse con la información disponible, en el caso en que no está ni siquiera agrupada por estratos de tamaño, consiste en estudiar el comportamiento histórico del desarrollo agrario por variables separadas y la forma en que se relacionan a lo largo del tiempo. Con este método se pueden hacer inferencias, por ejemplo, de la relación entre cambios tecnológicos, forma de tenencia de la tierra, uso del suelo y ocupación rural. A partir de este tipo de trabajos pueden elaborarse hipótesis acerca de la relación entre cambios producidos en la estructura agraria y dinámica poblacional. No es mucho lo que en este sentido puede realizarse, pues la índole de los datos puede falsear en buena medida las conclusiones aparentes de los movimientos de las variables a lo largo del tiempo. De todos modos, pueden constituir una vía de aprovechamiento de una cantidad de información disponible, por lo regular subutilizada, y complementarse con estudios como el que describiremos más adelante, que plantean criterios de agregación

9 Las cifras citadas provienen de cIDA, op. cit., cuadro 10, p. 25. 
de la información sobre la base de una tipología de relaciones de producción.

\section{AlgunAS REFLeXiones SOBRE EL PROCESO DE PRODUCCIÓN DE LOS DATOS}

Un problema fundamental es que el dato que utilizamos como materia prima en cualquier investigación no es "la realidad" directa que queremos investigar. Es algo que ha sido producido a partir de una indagación sobre esa realidad conducida de una manera determinada. Además, los resultados primarios de esas indagaciones han sido sujetos a un determinado nivel de manipuleo antes de pasar a su análisis. Cuando se trata de estudios con fuentes primarias tal manipuleo lo efectúa el investigador. No sucede lo mismo en los estudios de fuente censal.

A nuestro juicio, es muy correcto lo que señalan Manuel Castells y Emilio de Ipola: ${ }^{10}$ " . . . ninguna observación es realizable, sin una categorización que, si no es proporcionada por una teoría, procede necesariamente de la práctica institucional dominante, es decir, de las formas bajo las cuales las ideologías dominantes aprehenden prácticamente los fenómenos que se pretende observar...". En rigor, no hay dato que no sea construido y, en esta construcción, la teoría juega un papel indispensable y eminentemente productivo.

Si se trata, entonces, de considerar los datos como algo que se produce, resulta que buena parte de las dificultades con que se encuentran quienes desean investigar la estructura agraria a partir de considerar básica la determinación del tipo de relaciones de producción existentes en el agro, derivan de la forma en que se han construido los datos que se pretenden utilizar. Lo que desarrollamos a continuación es un criterio de construcción de datos agrarios de fuente censal, que permita estudiar, de manera precisa las relaciones de producción existentes. Es, de alguna manera, la forma de producir una materia semielaborada a partir de la cual, entre otras cosas, podrán estudiarse de manera provechosa las relaciones entre población y estructura agraria.

IV. Propuesta PARA ORganizar INFORMACIÓN AGROPECUARIA DE ORIGEN CENSAL, QUE REFLEJE LAS RELACIONES DE PRODUCCIÓN EN EL AGRO ${ }^{11}$

Antes de detallar los criterios propuestos para el agrupamiento de los datos, resulta necesario, si queremos ser consistentes con todo lo expre-

10 Manuel Castells y Emilio De Ipola, Práctica epistomológica y ciencias sociales, o cómo desarrollar la lucha de clases en el plano teórico sin internarse en la metafísica.

11 La metodología descrita es la utilizada en una investigación en proceso. 
sado antes, plantear cuáles son los "tipos de empresa" (delimitados de acuerdo a la forma de las relaciones de producción que en ellos se dan) que deseamos observar por separado.

\section{Subfamiliares}

En primer lugar, se tienen las explotaciones dirigidas por "campesinos pobres". Esto quiere decir, empresas agrarias cuyo producto no alcance a cubrir las necesidades del productor aceptadas por lo regular como mínimas. De aquí se infiere que por lo común estos campesinos deban recurrir de manera transitoria a la venta de su propia fuerza de trabajo en otras explotaciones rurales, o en otras actividades. Poseen un nivel de equipamiento inferior al medio.

\section{Familiares}

El grupo siguiente está dado por las empresas de tipo familiar, esto es, que no ocupan trabajadores asalariados permanentes, y que disponen de un nivel de equipamiento mínimo aceptable.

\section{Familiar-empresarial}

Luego consideramos aquellas explotaciones semejantes a las del grupo señalado antes, pero que utilizan en pequeña escala mano de obra asalariada permanente.

\section{Empresarial-familiar}

En este caso incluimos empresas semi-capitalistas, a las que consideramos separadas de las "totalmente" capitalistas por encontrar en las mismas un peso considerable de la fúerza de trabajo familiar dentro del total de mano de obra utilizada, aunque en ese total predomine la presencia de asalariados permanentes.

\section{Empresarial}

Este grupo está constituido por aquellas explotaciones en las que dominan las relaciones salariales en forma neta, o sea que pueden considerarse empresas capitalistas en sentido pleno.

En la medida en que la propiedad de la tierra genera situaciones diferentes en todos los tipos mencionados de explotaciones, se considera conveniente introducir, como criterio de clasificación, si la tierra ocupada es o no propiedad del productor. Con este agregado, llegamos a un total de 10 grupos en los que habría que clasificar la información, y cuya definición se basa en la forma de las relaciones de producción que aparece en cada empresa agraria.

Los criterios cuantitativos con base en los cuales se efectúa la agregación de la información, se basan en la tipología de empresas agrarias 
que se describió antes y en las características de los datos censales disponibles en Argentina.

En lo que hace al primer grupo, el subfamiliar, engloba a todas aquellas explotaciones en las que no se emplea mano de obra asalariada permanente y que no poseen ningún elemento motorizado en su equipamiento. Ni tractor, ni motocultivadoras, ni camión, ni automóvil. Como no se poseen datos sobre si el "productor" trabaja fuera de la explotación, se decidió tomar como referencia la existencia o no de algún equipo motorizado, ya que en el caso de Argentina parece ser un buen dato para diferenciar a los campesinos pobres en la actualidad.

El grupo siguiente, o sea el familiar, se define como constituido por todas las explotaciones que no emplean asalariados permanentes y que poseen algún equipo motorizado.

En el caso del grupo familiar-empresarial, se incluyen todas aquellas explotaciones en las que se contratan asalariados permanentes, pero en número inferior al de los no asalariados que trabajan (productor más familiares que trabajan).

Para definir las explotaciones incluidas en el grupo empresarial-familiar, se consideran todas las que utilicen mano de obra asalariada permanente en mayor número que la no asalariada, pero sin llegar a duplicarla.

Por último, el grupo empresarial queda constituido por las explotaciones en las que la mano de obra asalariada permanente es más del doble que la no asalariada.

Como ya se dijo, cada grupo así definido, se divide en dos, según sean o no propietarios de la tierra, con lo que se llega a un total posible de 10 grupos (posible, porque pueden haber grupos que en determinadas zonas no contengan ninguna explotación).

El criterio fundamental con el que delimitamos grupos de explotaciones es entonces el peso relativo de las relaciones salariales además del dato acerca de la propiedad o no propiedad de la tierra. Sin embargo, puede existir la necesidad de introducir criterios adicionales para efectuar cierta desagregación dentro de los grupos "básicos" definidos hasta ahora. Puede ocurrir que haya diferencias cuantitativas importantes dentro de algunos de los grupos, por ejemplo, en cuanto a la magnitud de la empresa. Por ese motivo se introduce la posibilidad de efectuar algunas desagregaciones adicionales, al tener en cuenta los ingresos de las explotaciones y el tamaño de las mismas; pero este tipo de clasificación no se puede definir antes de realizar la investigación, sino que es parte del proceso mismo de investigación. Lo único que interesa en este caso es explicitar la posibilidad de que sea a veces necesario definir nuevos grupos ad hoc.

Por otra parte, para que la información acerca de las estructuras agrarias pueda ser utilizada con provecho resulta indispensable una desagregación amplia a nivel regional. En el caso de Argentina, es posible 
disponer de datos a nivel de Departamento, que es la unidad administrativa de menor tamaño. El problema es que hay alrededor de quinientos departamentos en todo el país, por lo que resulta necesario, para poder analizar el conjunto de la información, realizar un agrupamiento de los mismos en regiones agrarias que tengan cierto grado de homogeneidad.

Esto es necesario, además, porque hay departamentos muy pequeños, y muy grandes, con lo cual no constituyen en sí mismos unidades comparables.

Esta tarea de regionalización no presenta cambios respecto de lo habitual en el tema. En nuestro trabajo, nos basamos en los tipos de uso del suelo y en el peso relativo de los distintos tipos de empresa agraria, para delimitar las zonas.

En lo que de manera específica hace a la utilización de la información de las características de la que se podrá disponer con una investigación de esta naturaleza, y el estudio de los movimientos de población, se pueden plantear dos tipos de enfoque. Por una parte, al observar los movimientos registrados en el pasado, que en zonas rurales se trata en general de expulsión de población, y si se dispone de información sobre la evolución de los niveles de las actividades rurales, se podrán sin duda elaborar hipótesis que expliquen esos movimientos en relación con el predominio de uno u otro tipo de empresa, o sea con el peso diferenciado de distinto tipo de relaciones de producción en diferentes zonas.

Por otra parte, se dispondrán de nuevos elementos como para "predecir" los movimientos futuros de población, teniendo en cuenta probables cambios futuros en los niveles de actividad, en la tecnología utilizada, etcétera.

\section{Características de InVestigaciones No baSADAS EN DATOS CENSALES}

No podríamos en esta oportunidad revisar la cuantiosa bibliografía que existe al respecto, pero sí referirnos a las líneas de orientación más importantes, en relación con su posible vinculación con investigacionès de fuente censal como la descrita en el punto anterior.

Por una parte, existen trabajos que describen distintos tipos de explotaciones rurales, y que tienen en cuenta las formas de las relaciones de producción en la empresa agraria. Los estudios de Antonio García ${ }^{12}$ y de Manuel Diéguez junior ${ }^{13}$ entran dentro de este tipo de trabajos.

Otro tipo de estudios, muy comunes, son aquellos basados en trabajos restringidos de encuestas a establecimientos rurales en determinadas

12 Antonio García, Reforma agraria y economía empresarial en América Latina, Santiago de Chile, Editorial Universitaria, 1967. Este es un libro, entre varios tel autor, donde se tratan los temas mencionados.

13 Manuel Diégues Junior, Establecimientos rurales en América Latina, Buenos Aires, Editorial Universitaria, 1967. 
áreas. En general, este tipo de trabajos ha producido hasta ahora resultados muy limitados, sin salir por lo regular de un estrecho marco empirista.

Hay otra clase de investigaciones, que entran dentro de la antropología social, en las que se profundiza mucho en el estudio de determinadas comunidades rurales y se relacionan los problemas económicos con los de la vida política, cultural, religiosa. ${ }^{14}$

En nuestra opinión, puede resultar de gran interés la posibilidad de complementación de una investigación con fuentes censales, con trabajos de la índole de los mencionados en último término.

No cabe duda, que cuando se dispongan de algunos resultados de la aplicación de los criterios metodológicos aquí descritos, se podrá replantear de nuevo el problema con los nuevos elementos que aporte la experiencia.

14 Un excelente trabajo que reúne estas características es el de Eduardo Archetti y Christianne Stolen, Los colonos del norte de Santa Fe, ni campesinos ni capitalistas, República Argentina, Universidad Nacional de Tucumán, 1974. 